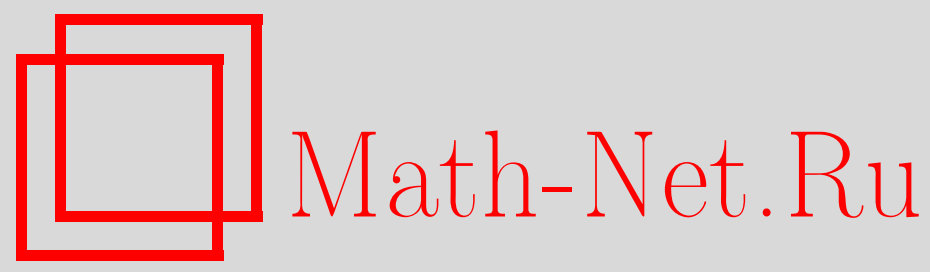

П. С. Геворкян, Шейповые морфизмы в транзитивные $G$-пространства, Матем. заметки, 2002, том 72, выпуск $6,821-827$

DOI: https://doi.org/10.4213/mzm469

Использование Общероссийского математического портала Math-Net.Ru подразумевает, что вы прочитали и согласны с пользовательским соглашением http://www.mathnet.ru/rus/agreement

Параметры загрузки:

IP : 54.89 .56 .158

26 апреля 2023 г., $15: 49: 21$ 


\title{
ШЕЙПОВЫЕ МОРФИЗМЫ \\ В ТРАНЗИТИВНЫЕ $G$-ПРОСТРАНСТВА
}

\author{
П. С. Геворкян
}

Во многих задачах теории шейпов важную роль играет следующая проблема: при каких условиях шейповый морфизм $F: X \mapsto Y$ топологического пространства $X$ в топологическое пространство $Y$ порождается некоторым непрерьвным отображением $f: X \mapsto Y$ ? В настоящей статье данная проблема рассматривается в эквивариантной теории шейпов и положительно решается для эквивариантно-шейповых морфизмов в транзитивные $G$-пространства, где $G$ - компактная группа со счетной базой. В качестве следствия доказывается достаточное условие равенства эквивариантных шейпов $G$-пространства $X$ и самой группы $G$. Доказаны также некоторые результаты, относящиеся к эквивариантным расслоениям, которые являются ключевыми для последних результатов и представляют самостоятельньй интерес.

Библиограффия: 5 названий.

1. Введение. Во многих задачах теории шейпов важную роль играет следующая проблема: при каких условиях шейповый морфизм $F: X \mapsto Y$ топологического пространства $X$ в топологическое пространство $Y$ порождается некоторым непрерьвньп отображением $f: X \mapsto Y$ ? Из построения теории шейпов непосредственно следует, что эта проблема имеет положительное решение в случае, когда $Y$ является ANR-пространством. В настоящей статье данная проблема рассматривается в эквивариантной теории шейпов и положительно решается для эквивариантно-шейповых морфизмов в транзитивные $G$-пространства, где $G$ - компактная группа со счетной базой. В качестве следствия доказьвается достаточное условие равенства эквивариантных шейпов $G$-пространства $X$ и самой группы $G$. Доказаны также некоторые результаты, относящиеся к эквивариантным расслоениям, которые являются ключевьми для последних результатов и представляют самостоятельньй интерес.

Действие группы $G$ на пространстве $X$ называется транзитивным, если орбита $G(x)=\{g(x): g \in G\}$ произвольной точки $x \in X$ совпадает с самим пространством $X$, т.е. если у $G$-пространства $X$ имеется ровно одна орбита. Примерами транзитивных $G$-пространств служат пространства смежных классов $\left.G\right|_{H}$ топологической групшы $G$ по ее замкнутьм подгрупам $H$ (на пространстве $\left.G\right|_{H}$ рассматривается действие группы $G$ посредством левых сдвигов: $\left.g\left(g^{\prime} H\right)=\left(g g^{\prime}\right) H\right)$. Более того, известно, что если действующая группа $G$ является компактной, то любое транзитивное $G$-пространство $X$ эквиморфно некоторому пространству смежных классов, а точнее пространству $\left.G\right|_{G_{x}}$, где $G_{x}=\{g \in G: g(x)=x\}$ - стационарная подгруппа (или стабилизатор) точки $x$. Последнее замечание позволяет в качестве транзитивных $G$-пространств рассматривать 
пространства смежных классов с естественным действием группы $G$, при этом не теряя общность рассуждений.

Другие факты теории непрерьвных групп преобразований можно найти в работах [1] и [2].

\section{2. Эквивариантные расслоения.}

ОПРЕДЕЛЕниЕ 1. Пусть $E$ и $B$ - произвольные $G$-пространства. Скажем, что эквивариантное отображение $p: E \mapsto B$ удовлетворяет аксиоме об эквивариантной накрывающей гомотопии относительно $G$-пространства $X$, если для любых эквивариантных отображений $f: X \mapsto E$ и $F: X \times I \mapsto B$ таких, что $F(x, 0)=p f(x), x \in X$, существует эквивариантное отображение $\widetilde{F}: X \times I \mapsto E$, для которого $\widetilde{F}(x, 0)=f(x)$ и $p \circ \widetilde{F}=F$, т.е. коммутативна диаграмма 1 .

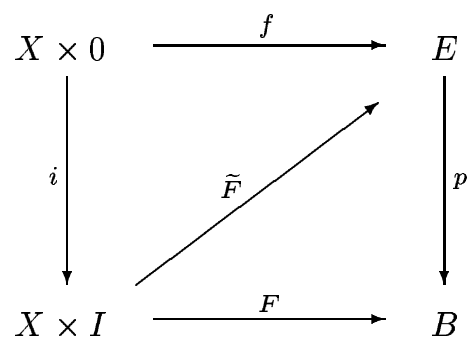

\section{ДиАГРАМмА 1}

ОПРЕДЕЛЕниЕ 2. Эквивариантное отображение $p: E \mapsto B$ называется әквивариантным расслоением, или $G$-расслоением, если оно удовлетворяет аксиоме об эквивариантной накрьвающей гомотопии относительно всех $G$-пространств.

ТЕОрема 1. Пусть $H$ - замкнутая подгруппа компактной группь Ли G. Тогда

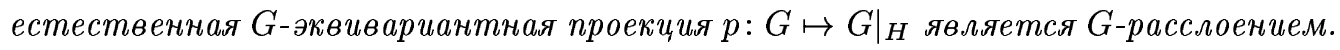

ДокАЗАтЕльСтво. Покажем, что $p:\left.G \mapsto G\right|_{H}$ удовлетворяет аксиоме об эквивариантной накрывающей гомотопии относительно всех $G$-пространств.

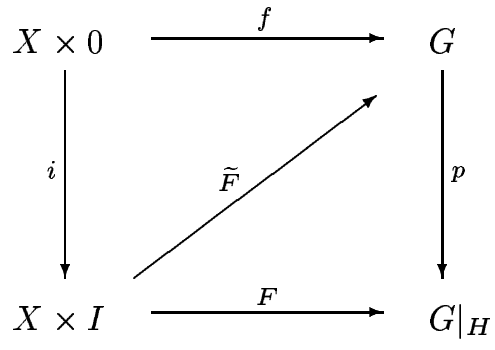

ДИАГРАММА 2

Пусть $X$-произвольное $G$-пространство, $f: X \mapsto G$-произвольное эквивариантное отображение, а $F: X \times\left. I \mapsto G\right|_{H}$ - эквивариантная гомотопия отображения $p \circ f: X \mapsto$ $\left.G\right|_{H}$, т.е. $F(x, 0)=p f(x)$ для любого $x \in X$. Требуется доказать существование такого 
эквивариантного отображения $\widetilde{F}: X \times I \mapsto G$, которое делает коммутативной диаграмму 2, или, что то же самое, вьполняются условия

$$
p \circ \widetilde{F}=F, \quad \widetilde{F}(x, 0)=f(x)
$$

для любого $x \in X$.

Так как $f$ - эквивариантное отображение, то $X=\left.X\right|_{G} \times G\left(\left.X\right|_{G} \times G\right.$ рассматривается как $G$-пространство с действием $g\left(\widetilde{x}, g^{\prime}\right)=\left(\widetilde{x}, g g^{\prime}\right)$ для произвольньх $g, g^{\prime} \in G$ и $\left.\left.\widetilde{x} \in X\right|_{G}\right)$. Следовательно, диаграмму 2 можно представить в виде, показанном на диаграмме 3.

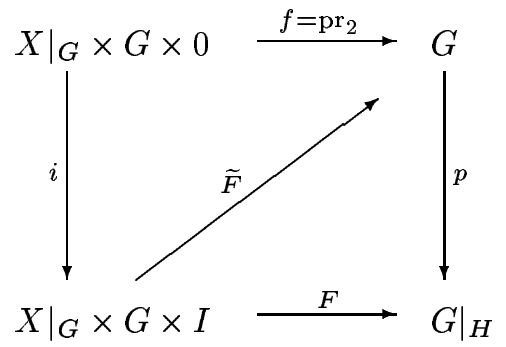

ДИАГРАММА 3

Рассмотрим диаграмму 4, где

$$
f_{e}=\left.f\right|_{\left(\left.X\right|_{G} \times\{e\} \times 0\right)}, \quad F_{e}=\left.F\right|_{\left(\left.X\right|_{G} \times\{e\} \times I\right)}, \quad i_{e}=\left.i\right|_{\left(\left.X\right|_{G} \times\{e\} \times 0\right)} .
$$

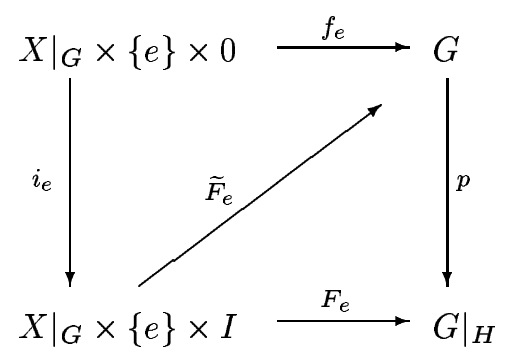

\section{ДиАГРАММА 4}

Так как $p:\left.G \mapsto G\right|_{H}-$ расслоение [3], то в последней диаграмме существует отображение $\widetilde{F}_{e}$, котороепреврашает всю диаграмму в коммутативную. Искомое отображение $\widetilde{F}:\left.X\right|_{G} \times G \times I \mapsto G$ определим формулой

$$
\widetilde{F}(\widetilde{x}, g, t)=g \widetilde{F}_{e}(x, e, t) .
$$

Эквивариантность отображения $\widetilde{F}$ проверяется непосредственно. Докажем коммутативность диаграммы 3 , что эквивалентно равенствам (см. (1))

$$
\widetilde{F}(\widetilde{x}, g, 0)=g \widetilde{F}_{e}(\widetilde{x}, e, 0)=g f_{e}(\widetilde{x}, e, 0)=g f(\widetilde{x}, e, 0)=f(\widetilde{x}, g, 0)
$$

для любого $\left.(\widetilde{x}, g, 0) \in X\right|_{G} \times G \times 0$,

$$
p \widetilde{F}(\widetilde{x}, g, t)=p\left(g \widetilde{F}_{e}(\widetilde{x}, e, t)\right)=g p \widetilde{F}_{e}(\widetilde{x}, e, t)=g \widetilde{F}_{e}(\widetilde{x}, e, t)=g F(\widetilde{x}, e, t)=F(\widetilde{x}, g, t)
$$

для любого $\left.(\widetilde{x}, g, t) \in X\right|_{G} \times G \times I$.

Тем самым, теорема доказана. 
ТеОРема 2. Пусть $H u K-$ замкнутые нормальные подгруппы группы $G u$ $H \subset K$. Если $\left.G\right|_{H}$ - группа Ли, то естественный $G$-әквивариантный әпиморфизм $p:\left.\left.G\right|_{H} \mapsto G\right|_{K}$ является $G$-расслоением.

ДокАЗАТЕльСтво. Покажем, что при заданных условиях $G$-эквивариантное отображение $p:\left.\left.G\right|_{H} \mapsto G\right|_{K}$ удовлетворяет аксиоме об эквивариантной накрывающей гомотопии относительно любого $G$-пространства $X$. Для этого достаточно доказать, что в диаграмме 5 , где $F: X \times\left. I \mapsto G\right|_{K}$ - эквивариантная гомотопия отображения $p \circ f: X \times\left. 0 \mapsto G\right|_{K}$, существует отображение $\widetilde{F}: X \times\left. I \mapsto G\right|_{H}$, делающее всю диаграмму коммутативной.

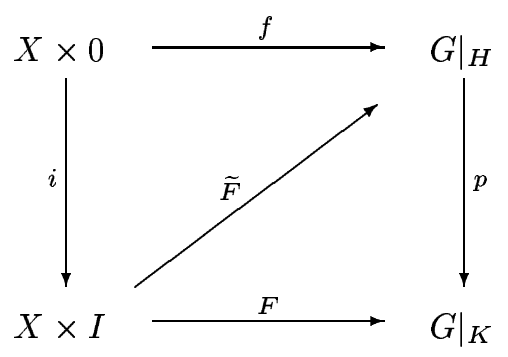

ДиАГРАмМА 5

В диаграмме 5 перейдем к пространствам $H$-орбит. Тогда получим диаграмму 6 , где $\pi_{0}$ и $\pi-H$-орбитные проекции, а $\left.f\right|_{H}$ и $\left.F\right|_{H}$ - отображения пространств $H$-орбит, порожденные эквивариантными отображениями $f$ и $F$ соответственно.

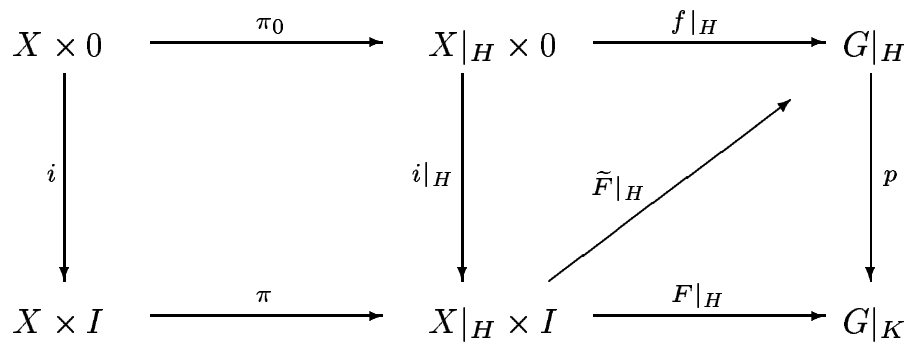

ДИАГРАММА 6

Заметим, что

$$
\left.f\right|_{H} \circ \pi_{0}=f,\left.\quad F\right|_{H} \circ \pi=F .
$$

Так как $H$ - замкнутая нормальная подгрупша групшы $G$, то на пространстве $\left.X\right|_{H} \times I$ действует группа $\left.G\right|_{H}$ по формуле

$$
\widetilde{g}(\widetilde{x}, t)=(g \widetilde{x}, t),
$$

где $\widetilde{g}=\left.g H \in G\right|_{H},(\widetilde{x}, t)=\left.(H x, t) \in X\right|_{H} \times I$, a $(g \widetilde{x}, t)=\left.(H g x, t) \in X\right|_{H} \times I$. Имеется и естественное действие группы $\left.G\right|_{H}$ на $\left.G\right|_{K}$, так как

$$
\left.G\right|_{K}=\left.\left(\left.G\right|_{H}\right)\right|_{\left(\left.K\right|_{H}\right)},
$$


где $\left.K\right|_{H}$ - замкнутая подгрупша компактной групшы Ли $\left.G\right|_{H}$. Таким образом, компактная група Ли $\left.G\right|_{\text {н }}$ действует на всех пространствах левой части диаграммы 6 и все

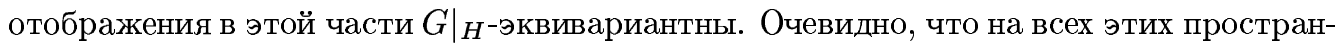
ствах действует и группа $G$ (через естественный эпиморфизм $\left.G \mapsto G\right|_{H}$ ). Очевидно, что все отображения диаграммы 6 также и $G$-эквивариантны.

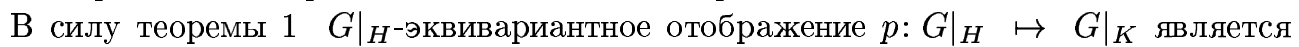
$\left.G\right|_{H}$-расслоением. Следовательно, в диаграмме 6 существует $\left.G\right|_{H}$-эквивариантная гомотопия $\left.\widetilde{F}\right|_{H}:\left.X\right|_{H} \times\left. I \mapsto G\right|_{H}$, делающая правую часть этой диаграммы коммутативной. Теперь искомую $G$-эквивариантную гомотопию $\widetilde{F}: X \times\left. I \mapsto G\right|_{H}($ см. диаграмму 5$)$ можно задать формулой

$$
\widetilde{F}=\left.\widetilde{F}\right|_{H} \circ \pi \text {. }
$$

Проверим $G$-эквивариантность отображения $\widetilde{F}$ :

$$
\widetilde{F}(g(x, t))=\left.\widetilde{F}\right|_{H} \pi(g(x, t))=\left.\widetilde{F}\right|_{H}(g \pi(x, t))=\left.g \widetilde{F}\right|_{H} \pi(x, t)=g \widetilde{F}(x, t) .
$$

Заметим, что

$$
\widetilde{F}(x, 0)=\left.\widetilde{F}\right|_{H} \pi(x, 0)=\left.f\right|_{H} \pi(x, 0)=\left.f\right|_{H} \pi_{0}(x, 0)=f(x, 0) .
$$

И, наконец, $p \circ \widetilde{F}=\left.p \circ \widetilde{F}\right|_{H} \circ \pi=\left.F\right|_{H} \circ \pi=F$.

Теорема доказана.

3. $G$-шейповые морфизмы, порожденные эквивариантными отображениями.

Теорема 3. Пусть $G$ - компактная группа со счетной базой, $H \subset G$ - замкнутая нормальная подгруппа, а $X$ - произвольное $G$-пространство. Тогда любой $G$-шейповый морфизм $F:\left.X \mapsto G\right|_{H}$ порожсдается некоторым әквивариантным отображением.

ДокАзАтЕльство. Так как группа $G$ компактна и имеет счетную базу, то такова и группа $\left.G\right|_{H}$. По известной теореме Понтрягина (см. [4, теорема 68, с. 332]) существуют замкнутые нормальные делители $K_{i}, i=1,2, \ldots$, групшы $\left.G\right|_{H}$ такие, что $K_{i+1} \subset K_{i}$, а $\left.\left(\left.G\right|_{H}\right)\right|_{K_{i}}-$ группа Ли для любого $i=1,2, \ldots$ и

$$
\left.G\right|_{H}=\lim _{\longleftarrow}\left\{\left.\left(\left.G\right|_{H}\right)\right|_{K_{i}} ; p_{i, i+1}\right\},
$$

где $p_{i, i+1}:\left.\left.\left(\left.G\right|_{H}\right)\right|_{K_{i+1}} \mapsto\left(\left.G\right|_{H}\right)\right|_{K_{i}}-$ естественные эпиморфизмы, порожденные включениями $K_{i+1} \subset K_{i}$.

Заметим, что для любого $i=1,2, \ldots$ группа $\left.\left(\left.G\right|_{H}\right)\right|_{K_{i}}$ изоморфна (топологически и алгебраически) группе $\left.G\right|_{\widetilde{K}_{i}}$, где $\widetilde{K}_{i}=p^{-1}\left(K_{i}\right)$, а $p:\left.G \mapsto G\right|_{H}$ - естественный эпиморфизм. Групшы $\widetilde{K}_{i}, i=1,2, \ldots$, будучи непрерывными прообразами замкнутых нормальных подгрупп $\left.K_{i} \subset G\right|_{H}$, замкнуты и нормальны. Таким образом,

$$
\left.G\right|_{H}=\lim _{\longleftarrow}\left\{\left.G\right|_{\widetilde{K}_{i}} ; p_{i, i+1}\right\},
$$

где все отображения $p_{i, i+1} G$-эквивариантны, если пространства $\left.G\right|_{\widetilde{K}_{i}}$ рассматривать с естественным действием групшы $G$. 
Пусть теперь дан $G$-шейповьй морфизм $F:\left.X \mapsto G\right|_{H}$. Рассмотрим $G$-шейповое отображение $S_{G}\left(p_{i}\right) \circ F:\left.X \mapsto G\right|_{\widetilde{K}_{i}}$, где $S_{G}-G$-шейповьй функтор, а $p_{i}:\left.G \mapsto G\right|_{\widetilde{K}_{i}}-$ предельные проекции. Так как все $\left.G\right|_{\widetilde{K}_{i}}$ суть $G$-ANR-пространства, то существуют $G$-эквивариантные отображения $f_{i}:\left.X \mapsto G\right|_{\widetilde{K}_{i}}$ такие, что

$$
S_{G}\left(p_{i}\right) \circ F=S_{G}\left(f_{i}\right)
$$

Ho

$$
\begin{aligned}
S_{G}\left(f_{i}\right) & =S_{G}\left(p_{i}\right) \circ F=S_{G}\left(p_{i, i+1}\right) \circ S_{G}\left(p_{i+1}\right) \circ F \\
& =S_{G}\left(p_{i, i+1}\right) \circ S_{G}\left(f_{i+1}\right)=S_{G}\left(p_{i, i+1} \circ f_{i+1}\right) .
\end{aligned}
$$

Итак, $G$-отображения $f_{i}$ и $p_{i, i+1} \circ f_{i+1}$ в $G$-ANR-пространство $\left.G\right|_{\widetilde{K}_{i}}$ порождают один и тот же $G$-шейповый морфизм. Значит, они $G$-гомотопны:

$$
f_{i} \underset{G}{\sim} p_{i, i+1} \circ f_{i+1}
$$

Положим $h_{1}=f_{1}$. В силу теоремы 2 все отображения $p_{i, i+1}-G$-расслоения. Следовательно, существует $G$-отображение $h_{2}:\left.X \mapsto G\right|_{\widetilde{K}_{2}}$ такое, что $h_{2} \underset{G}{\simeq} f_{2}$ и $h_{1}=p_{1,2} \circ h_{2}$. Продолжая это построение по индукции, получим $G$-отображения $h_{i}:\left.X \mapsto G\right|_{\widetilde{K}_{i}}$ такие, что

$$
h_{i} \underset{\bar{G}}{\widetilde{f}} f_{i}, \quad h_{i}=p_{i, i+1} \circ h_{i+1}
$$

Положим

$$
h=\lim _{\longleftarrow} h_{i} .
$$

Очевидно, что $h-G$-отображение из $X$ в $\left.G\right|_{H}$. Докажем, что $h$ искомое, т.е.

$$
S_{G}(h)=F \text {. }
$$

В самом деле, в силу непрерьвности шейпового функтора [5] имеем

$$
S_{G}(h)=S_{G}\left(\lim _{\longleftarrow} h_{i}\right)=\lim _{\longleftarrow} S_{G}\left(h_{i}\right)=\lim _{\longleftarrow} S_{G}\left(f_{i}\right)=\lim _{\longleftarrow} S_{G}\left(p_{i}\right) \circ F=1 \circ F=F .
$$

Теорема доказана.

ТеОрема 4. Пусть $G$ - компактная группа со счетной базой, $H \subset G-з а-$ мкнутая нормальная подгруппа, а $X$ - произвольное $G$-пространство. Если существует $G$-шейповый морфизм $F:\left.X \mapsto G\right|_{H}$, то $X=G \times_{H} A$, где $A$ - некоторое $H$-пространство, а $F$ порохсден $G$-әквивариантным отображсением $f: G \times_{H} A \mapsto$ $\left.G\right|_{H}$, заданным формулой $f([g, a])=g H$.

ДокАЗАТЕЛЬСТво. Пусть $F:\left.X \mapsto G\right|_{H}-G$-шейповый морфизм. По теореме 3 существует $G$-эквивариантное отображение $f:\left.X \mapsto G\right|_{H}$ такое, что $S_{G}(f)=F$. Положим $A=f^{-1}(e H)$. Известно [2], что тогда $X=G \times_{H} A$, a $f([g, h])=g H$.

В формулировке последней теоремы, взяв в качестве замкнутой нормальной подгруппы $H$ единичную группу $\{e\}$, получим 
СлЕДСтвиЕ 1. Если существует $G$-шейповый морфизм $F$ из $G$-пространства $X$ в компактную группу $G$ со счетной базой, то $X=\left.X\right|_{G} \times G$, a $F$ порожден естественной проекиией $\mathrm{pr}_{2}:\left.X\right|_{G} \times G \mapsto G$.

Теорема 5. Если существует $G$-иейповый морфизм из $G$-пространства $X$ в компактную группу $G$ со счетной базой $u \operatorname{sh}\left(\left.X\right|_{G}\right)=0, \operatorname{mosh}_{G} X=\operatorname{sh}_{G} G$.

ДокАЗАтЕльСтво. Согласно следствию $1 X=\left.X\right|_{G} \times G$, где на пространстве $\left.X\right|_{G} \times G$ групша $G$ действует формулой $g\left(\widetilde{x}, g^{\prime}\right)=\left(\widetilde{x}, g g^{\prime}\right)$ для любых $\left.\left(\widetilde{x}, g^{\prime}\right) \in X\right|_{G} \times G$ и $g \in G$. Вычислим

$$
\operatorname{sh}_{G}\left(\left.X\right|_{G} \times G\right)=\operatorname{sh}_{G}\left(\left.X\right|_{G}\right) \times \operatorname{sh}_{G} G=0 \times \operatorname{sh}_{G} G=\operatorname{sh}_{G} G .
$$

\section{СПИСОК ЦИТИРОВАННОЙ ЛИТЕРАТУРЫ}

[1] Palais R.S. The classification of $G$-spaces // Mem. Amer. Math. Soc. 1960. №36. P. 1-72.

[2] Бредон Г. Введение в теорию компактных групп преобразований. М.: Наука, 1980.

[3] Gleason A. Spaces with a compact Lie group transformations // Proc. Amer. Math. Soc. 1950. V. 1. № 1. P. 35-43.

[4] Понтрягин Л. С. Непрерывные группы. М.: Гостехиздат, 1954.

[5] Holsztynski W. Continuity of Borsuk's shape functor // Bull. Acad. Polon. Ski. 1971. V. 19. №12. P. 1105-1108.

Московский государственный университет им. М.В. Ломоносова E-mail: gevorgyan@mech.math.msu.su, pgev@angelfire.com 